Didáctica Geográfica n 22, 2021, pp. 199-219

DOI: https://doi.org/10.21138/DG.631

ISSN electrónico: 2174-6451

\title{
MIRADAS URBANAS EN LA FORMACIÓN INICIAL DEL PROFESORADO
}

\author{
URBAN PERSPECTIVES IN TEACHER EDUCATION
}

REGARDS URBAINS DANS LA FORMATION INITIALE DES ENSEIGNANTS

Rosa M. Medir Huerta

Universitat de Girona

rosa.medir@udg.edu

Anna Serra-Salvi
Universitat de Girona
anna.serra@udg.edu

Recibido: 25/05/2021

Aceptado: 24/06/2021

\section{RESUMEN:}

Una asignatura obligatoria en la formación de maestros y maestras de educación primaria en la Universidad de Girona se desarrolla a partir de temas de Geografía social en relación con los Objetivos de Desarrollo Sostenible (ODS). Explicitamos, en este artículo, el trabajo realizado de geografía urbana, desde los Objetivos 5 (Igualdad de género) y 11 (Ciudades y comunidades sostenibles). Se presentan cuatro actividades realizadas con los y las estudiantes que buscan mirar la ciudad de una forma alternativa y coherente con los ODS mencionados. A la vez, creemos que dichas miradas son útiles y formativas para una ciudadanía crítica y más aún, para futuros docentes. La imagen de la ciudad, su mirada en clave de género, el espacio público y la visión futura de la ciudad ideal son los temas que 
se tratan en sesiones de clase, tanto en el aula como a través de trabajo de campo en el exterior. Los resultados demuestran que estos planteamientos resultan novedosos y útiles para los estudiantes para desarrollar conocimiento sobre la ciudad y la sostenibilidad.

\section{Palabras clave:}

Ciudad; Espacio público; Género; Formación de maestros; ODS.

\section{Asbract:}

A compulsory subject in the training of primary education teachers is developed along social geographic themes inherent, currently, to the Sustainable Development Goals. In this article we explain the work carried out on urban geography, from Objectives 5 (Gender equality) and 11 (Sustainable cities and communities). Four activities carried out with students who seek to see the city in an alternative way and consistent with the aforementioned SDGs are presented. At the same time, we believe that such views are useful and formative for critical citizenship and even more so, for future teachers. The image of the city, the city seen from a gender perspective, the public space in the city and the ideal city are the topics covered in class sessions in the classroom and field work abroad. The results demonstrate new approaches for students about the knowledge of the city and the concept of sustainability.

\section{KeY WORDS:}

City; Public space; Gender; Teacher education; GDS.

\section{RÉSUMÉ:}

Une matière obligatoire dans la formation d'instituteurs d'enseignement primaire se développe tout au long de sujets géographiques sociaux inhérents actuellement aux Objetifs de Développement Durable. Dans cet article, nous explicitons le travail réalisé de géographie urbaine, du point de vue des Objectifs 5 (Égalité des genres) et 11 (Villes et communautés durables). Nous présentons quatre activités réalisées avec les étudiants les qui cherchent à regarder la ville d'une façon alternative et cohérente avec les ODD cités. En même temps, nous pensons que ces regards sont utiles et formatifs pour une population critique et, davantage, pour de futurs enseignants. L'image de la ville, la ville vue du point de vue des genres, l'espace public dans la ville et la ville idéale sont les sujets traités en séances de cours en salle de classe et de travail sur le terrain à l'extérieur. Les résultats montrent de nouvelles approches pour les étudiants/es à propos de la connaissance de la ville et du concept de durabilité.

\section{Mots CLÉS:}

Ville; espace public; genres; formation d'instituteurs; ODD. 


\section{INTRODUCCIÓN}

En la formación inicial del profesorado de Educación Primaria se incluyen unas horas formativas en Didáctica de las Ciencias Sociales, en las cuáles seleccionamos los contenidos y las actividades posibles a desarrollar con los estudiantes universitarios del Grado de Educación Primaria. En la Universidad de Girona, la Didáctica de la Geografía y la Didáctica de la Historia son las disciplinas desde donde emanan las guías docentes que suman trece créditos ECTS (diez créditos para un módulo de Didáctica de las Ciencias Sociales y tres créditos para una asignatura llamada Sociedad y Sostenibilidad). Situémonos en ésta última asignatura. La finalidad principal de dicha asignatura se centra en profundizar en el concepto de sostenibilidad, especialmente desde el punto de vista social. Los primeros años, lo hacíamos para desembocar en el concepto de educación para la sostenibilidad. Pero, más recientemente, desde la irrupción de los Objetivos del Desarrollo Sostenible (ODS) de la Agenda 2030, hemos decidido reorientar nuestro programa docente para trabajar dichos objetivos y relacionarlos con la educación para la ciudadanía sostenible (Serra-Salvi y Medir, 2021). La educación para la sostenibilidad forma parte de una mención (asignaturas optativas), a la cual acuden algunos estudiantes del Grado.

Los ODS aparecen, hoy en día, como una novedad para algunas materias, o bien como una redirección necesaria de tantas otras; por ejemplo, en las universidades, profesorado de todas las áreas de conocimiento se sienten atraídos y comparten una nueva orientación de sus disciplinas hacia los ODS. ${ }^{1}$ Pensamos que los ODS no son una novedad para la Geografía, prácticamente todos ellos son temas geográficos que han sido trabajados siempre en los currículos geográficos. Hoy, la Geografía se orienta hacia los ODS, pero cuando no existían los ODS, simplemente era Geografía, y después de 2030, seguirá siendo Geografía. A este respecto, la Asociación de Geografía de España se pronunció en estos términos:

"Que en el contexto actual de desarrollo de la humanidad de la problemática ambiental existente a escala planetaria y de obligado cumplimiento de los Objetivos de Desarrollo Sostenible, establecidos por las Naciones Unidas, debe recordarse que las líneas maestras de estos ODS coinciden, de forma casi absoluta, con los contenidos y competencias desarrollados por la disciplina geográfica” (AGE, 2019)

\footnotetext{
1 Como ejemplo, en la Universidad de Girona se ha creado una Red de innovación docente, amparada por el Instituto de Ciencias de la Educación (ICE) de la propia universidad, en la cual participa profesorado de la mayoría de facultades con el objetivo de compartir y actualizar la docencia hacia el trabajo de los ODS. Las autoras de este artículo pertenecemos a dicha red.
} 
Mucho antes, en 2007, la Declaración de Lucerna sobre Educación Geográfica para el Desarrollo Sostenible ya dejaba claro que los temas que la Década de la Educación para el Desarrollo Sostenible 2005-2014 (lanzada por las Naciones Unidas) "incluyendo medio ambiente, agua, desarrollo rural, consumo sostenible, turismo sostenible, comprensión intercultural, diversidad cultural, cambio climático, reducción de desastres, biodiversidad y economía de mercado, tienen una dimensión geográfica. Esta Declaración propone que el paradigma del desarrollo sostenible sea integrado en la enseñanza de la Geografía en todos los niveles y en todas las regiones del mundo" (Haubrich, Reinfried y Schleicher, 2007)

La Geografía sirve para comprender el mundo global, más que cualquier otra disciplina. Colaborativamente con la historia, las dos ciencias sociales presentes con fuerza en la educación obligatoria han de dirigir el aprendizaje de una ciudadanía global. Para ello, la Didáctica de la Geografía también debe renovar o actualizar sus planteamientos. Los currículos nacionales, a menudo, no lo facilitan, según Rafael de Miguel $(2018$, 48) "el currículo español se ha quedado desfasado, y debido a su rigidez no es capaz de orientar procesos de enseñanza y aprendizaje de los verdaderos contenidos geográficos que explican la organización social del mundo actual desde un enfoque espacial". Tras un análisis comparativo de diferentes currículos con el caso español, el mismo autor propone una propuesta curricular basada en seis enfoques contemporáneos, para la enseñanza de la geografía escolar, adecuados al modelo de formación docente -inicial y continua- así como a la permanente y necesaria innovación en las aulas. Uno de estos enfoques es, sin duda, la educación geográfica para el desarrollo sostenible.

La concepción de la Geografía en todos los grandes documentos internacionales del siglo XX y XXI redactados por las asociaciones internacionales de la enseñanza de la Geografía (Unión Geográfica Internacional -UGI y otras) ha ido evolucionando hacia la globalidad. La última carta lanzada por la UGI, Declaración Internacional sobre Educación Geográfica de 2016, nos demuestra la contribución de la educación geográfica para la comprensión de desafíos globales como el desarrollo sostenible, el cambio climático, la globalización -económica, cultural y social en global debido a los avances en las tecnologías de transporte y comunicación- o el progreso del multilatelarismo y la geopolítica global después del fin de la guerra fría (De Miguel, 2020).

En el ámbito universitario se están llevando a cabo experiencias para la implementación de los ODS con diversidad de enfoques académicos. Desde los que proponen la incorporación de los contenidos totales o parciales de los actuales ODS a las competencias transversales en el ámbito de la formación de profesorado, aún antes de su declaración (Vilches y Gil, 2012), hasta la perspectiva de una educación transformadora liderada por el profesorado en sus propias y distintas asignaturas que, habiendo recibido una formación previa, se lanzan a cambiar y adaptar sus objetivos y metodologías docentes (Collazo y Granados, 2020) 
En los niveles de la educación obligatoria también surgen equipos de profesorado que tratan de responder al reto de incorporar los ODS en los procesos de enseñanza y aprendizaje. Como en el caso anterior, desde mucho antes de la declaración de los ODS, Granados (2010) explora en diversos trabajos el interés que suscita la cuestión del desarrollo sostenible para la enseñanza de la Geografía en la educación secundaria. Recientemente, otros estudios realzan el papel de las ciencias sociales en secundaria para vincular y demostrar su relación con los ODS (Corrales, M. et al., 2020) y de forma muy concreta, cabe destacar la propuesta la introducción de los ODS de modo transversal y de forma gamificada en los contenidos de geografía de $1^{\circ}$ de ESO (Corrales y Garrido, 2021).

Las autoras nos situamos en estos ámbitos de la Didáctica de la Geografía y decidimos dedicar un tiempo importante de los tres créditos de docencia a la comprensión de la ciudad desde diferentes miradas vinculadas a dos Objetivos del Desarrollo Sostenible.

\section{DESARROLLO DE CUATRO ACTIVIDADES SOBRE LA CIUDAD}

\subsection{Miradas urbanas en nuestra propuesta docente}

El mundo urbano es cada vez más presente en nuestras formas de vida, y aunque nuestros estudiantes de una universidad situada en una ciudad mediana de Catalunya, a veces, no tienen conciencia de vivir en una ciudad - por sus procedencias geográficaspensamos que no es exactamente así, ya que, preguntando espontáneamente por sus hábitos de movilidad, comerciales, de ocio, constatamos que el proceso de urbanización se extiende en todo el territorio de influencia de la Universidad de Girona. Por ello y porque, en el futuro, más personas vivirán en las ciudades -en 2030, 5000 millones de personas- nos interesa tratar desde diferentes puntos de vista, aspectos geográficos de la ciudad en relación al Objetivo 11 (Ciudades y comunidades sostenibles) al cual unimos aspectos relacionados con el género y el urbanismo (Objetivo 5, Igualdad de género). Entendemos que, actualmente, el futuro de una ciudad sostenible e igualitaria pasa por integrar las cuestiones de género en su planificación. Cuando diseñamos la asignatura nos pareció de suma importancia poner luz a dicho asunto. Nuestra formación como geógrafas sumada a la educación para la sostenibilidad nos lleva a querer colaborar con el empoderamiento de las personas -en este caso, futuros docentes- para la transformación social. Dotar a los estudiantes de información y conocimiento es el primer paso para que estos adquieran la mirada crítica para provocar un cambio social, como ciudadanos y como docentes, los cuales tienen en sus manos la transmisión de valores y la formación de una ciudadanía global, o sea reflexiva, crítica, competente y comprometida. Nos preguntamos: ¿cómo podrá nuestro alumnado implicarse en la planificación urbana de la ciudad? Los docentes, ¿deben y pueden aportar su mirada educativa para diseñar 
urbanísticamente la ciudad? Creemos que sí; para ello hemos programado las diferentes actividades que ahora explicitamos.

Hemos seleccionado cuatro actividades en relación a la ciudad que pensamos pueden dar formación y pautas de actuación como ciudadanos y profesores comprometidos a nuestros estudiantes actuales, mañana profesores en ejercicio. Son las siguientes:

1) La imagen de su ciudad, o el plano mental de la ciudad que más conocen. Siguiendo las ideas instauradas por Kevin Lynch (1960) en los años sesenta del siglo pasado y que tanto han servido a urbanistas y docentes que parten del mapa mental para el conocimiento de la ciudad con sus alumnos.

2) Enseñar a leer la ciudad en clave de género, o interpretar la vida en la ciudad desde una perspectiva de género. Para ello partimos de las ideas descritas por geógrafas y urbanistas que han investigado acerca de los usos distintos de los espacios en las ciudades según género (Bofill, 1993; Ortiz et al., 2014).

3) Analizar el nivel de sostenibilidad (ámbito social) del espacio público, siguiendo los conceptos clave que permiten interpretar las relaciones de los ciudadanos con los espacios públicos (Serra-Salvi, 2019).

4) Redacción acerca de "la ciudad que quiero", a modo de conclusión: unas pocas líneas que nos pueden servir para detectar si los aspectos que las profesoras hemos querido destacar se han incorporado en el pensamiento de los estudiantes.

Con el conjunto de las cuatro actividades estamos poniendo en práctica una actuación docente coherente con la educación para la sostenibilidad como la descrita por Tilbury (2011), especialmente referente a aprender a formular preguntas críticas, aclarar los propios valores, plantearse futuros más positivos y sostenibles, aprender a pensar en modo sistémico y aprender a responder a través del aprendizaje aplicado.

\subsection{La imagen de la ciudad}

La primera actividad, introductoria, de la ciudad consiste en dibujar el mapa mental de su ciudad (también de un pueblo) a través de sencillas indicaciones que asimilan a las que dejó establecidas el urbanista norteamericano Kevin Lynch en los años sesenta. Para comprender el espacio urbano, Lynch (1960) usaba las imágenes urbanas de los ciudadanos.

Sus trabajos acerca de la forma de la ciudad y los estudios relacionados con las percepciones humanas de la ciudad y cómo deberían afectar el diseño de la ciudad, fueron muy influyentes en la época y siguen interesando a geógrafos, urbanistas y profesores de geografía. Hoy en día, en la época de las tecnologías de la información geográficas, 
se siguen realizando experiencias pedagógicas usando lápiz y papel para estudiar y comprender eficientemente el territorio y la ciudad (Khusnutdinova et al., 2019).

Según Lynch (2015), la imagen urbana se conforma de una serie de elementos que hacen de un lugar o espacio identificable, con el usuario, con un grupo social, con alguna actividad o con la misma población residente. Así, un espacio público, lugar o ciudad con buena imagen urbana, deberá contar con algunos elementos que darán una mejor legibilidad al espacio urbano. La legibilidad, es un atributo para Lynch, de los lugares. Esta legibilidad, sirve como un elemento que potencia la correcta organización y orientación de un usuario o población residente o ajena al lugar. Una ciudad legible será aquella en la cual sus residentes o usuarios identifiquen, estructuren y den significado a los lugares frecuentados. Si asimilamos "legibilidad" a "comprensión" obtenemos el interés especial que esta forma de ver la ciudad tiene para el profesorado.

Para organizar el análisis de los mapas mentales, Lynch (2015) estableció cinco elementos comunes que aparecen en todos los dibujos de la ciudad, hayan sido realizados por mayores o por jóvenes. Se trata de: 1) sendas, los canales a través de los cuáles el observador se mueve; 2) bordes, las fronteras entre dos lugares; 3 ) distritos o barrios, las secciones de la ciudad con aspectos bidimensionales y un carácter común; 4) nodos, o los puntos de la ciudad que son el foco del tránsito; 5) hitos, los objetos que se pueden ver desde varios ángulos y distancias.

Veremos, en los resultados, un acercamiento de la visión de las ciudades ("sus ciudades") que tienen nuestros estudiantes.

\subsection{La ciudad en clave de género}

Para introducirnos en una mirada de género a la ciudad hemos diseñado dos etapas: en la primera, una actividad en clase, de adquisición de conceptos básicos y de reflexión sobre la propia experiencia. En la segunda, un trabajo de campo de observación de la ciudad, así como de contraste con los conocimientos adquiridos y análisis crítico sobre la percepción personal de la realidad.

La actividad de aula se lleva a cabo a partir de un blog preparado por las profesoras (https://ciutatigenere.blogspot.com). El ejercicio se estructura en tres partes secuenciadas. La primera parte es una encuesta individual para que el estudiantado tome conciencia de su propia percepción acerca de la vida de las mujeres y de la violencia de género en la ciudad. Las preguntas tratan de la presencia de esta violencia en el espacio público, "micro violencias" o posibles presiones hacia las mujeres desde el urbanismo. Esa misma encuesta individual se vuelve a responder una vez realizada la parte central de la actividad, permitiendo al alumnado constatar su evolución en relación con el tema introducido. La parte central cuenta con tres actividades a realizar en grupos de tres estudiantes. En cada 
tarea se presentan dos lecturas distintas y un vídeo/documental breve sobre los temas más clásicos de género y ciudad. Dicho material quiere establecer una base de conocimientos esenciales sobre el tema, a fin de que los estudiantes puedan participar de manera crítica y activa en los debates. El diálogo entre el alumnado se genera a partir de preguntas reflexivas y críticas al final de cada tarea. Se trata de cuestiones que parten de la propia experiencia del alumnado y de observaciones de su entorno más cercano; se busca confrontar la información adquirida en los textos leídos o documentales vistos con la realidad vivida del propio alumno y hacerles reflexionar sobre sus propias actitudes. Las temáticas escogidas presentes en los textos y filmaciones son las siguientes: introducción al urbanismo y género (con especial incidencia en los transportes); dinámicas de género en la ciudad; tareas de atención a las personas y el espacio urbano; el tiempo y la ciudad; los espacios de miedo; las mujeres sin hogar en el espacio público.

En la segunda etapa, el trabajo de campo se lleva a cabo en equipos y en el marco de una ciudad o de un municipio grande a escoger. Se estructura en tres partes. La primera trata de las representaciones de la mujer en el espacio urbano. Para ello, los estudiantes deben callejear e ir observando, tomando notas y fotografías de todas aquellas representaciones de la mujer que descubren en los escaparates, esculturas, grafitis, dibujos en las paredes, señales... Se les pide que elaboren una ficha para cada representación en la que conste una fotografía, el formato en que se encuentra la representación (escultura, póster, cartel...) y una descripción de las características y/o valores que ellos o ellas consideren que se le atribuyen a la mujer figurada. A continuación, redactan unas conclusiones. La segunda parte del trabajo de campo trata los roles que llevan a cabo hombres y mujeres. Para ello, se pide al alumnado observar: dos supermercados, dos puestos del mercado, dos plazas públicas, dos autobuses, dos bares, una biblioteca y la entrada o salida de una escuela. Con relación a esta observación deben tomar nota de cuántos hombres y cuántas mujeres hay y que están haciendo. Finalmente, la tercera parte del trabajo son las conclusiones acerca de las observaciones hechas. En ellas se debe reflexionar/discutir de manera crítica acerca de los roles y los valores que esconden, así cómo se transmiten en el espacio público. Se invita a los estudiantes a ofrecer propuestas para la construcción de una ciudad igualitaria.

\subsection{El espacio público de la ciudad}

Con la voluntad de trabajar el objetivo número 11 de la Agenda 2030 acerca de la construcción de ciudades y comunidades sostenibles, una de las prácticas de la asignatura consiste en el análisis de la sostenibilidad (ámbito social) en los espacios públicos. Nos inspira la idea Borja (2013) cuando nos dice que la ciudad es antes que nada espacio público y el espacio público es la ciudad. 
El espacio público es el espacio de la ciudadanía por excelencia, el espacio propio de las relaciones y las iniciativas entre grupos, los intercambios, una zona de estímulo, algo más que un simple lugar de tránsito de un sitio a otro (Lefebvre 1978). Se configura un tejido urbano concreto, de mujeres y de hombres, de niños, de jóvenes y de personas mayores en función de las posibilidades de desarrollar la vida que el espacio público ofrece. No en vano, el espacio urbano es la expresión propia de los valores que identifican la comunidad que lo rodea. Como afirma Tello (2002, p.16) "el espacio público define la calidad de la ciudad, porqué indica la calidad de vida de la gente y la calidad de la ciudadanía de sus habitantes". En este sentido, el concepto de sostenibilidad en relación a la ciudad coincide con el concepto de calidad de vida.

Consideramos el nivel escalar de barrio y -en todo caso- la concreción de una plaza o un parque público los ámbitos idóneos en los cuales valorar los elementos que aportan calidad de vida a los vecinos, y a la ciudadanía en general. Efectivamente, el parámetro de sostenibilidad en la ciudad contempla muchos aspectos que tienen que ver con las viviendas, la accesibilidad universal, el transporte, los roles económicos, las desigualdades sociales, la ausencia o la presencia de conflictos, etc.

Hemos observado que, con frecuencia, los estudiantes dan al concepto de sostenibilidad un enfoque mayormente ambientalista, es decir, piensan en el cambio climático, la gestión de los residuos y la atención a la biodiversidad, olvidándose de los valores sociales que dan sentido pleno al término. El concepto de sostenibilidad, más allá de los importantes valores de la ecología, integra los valores de justicia social, diversidad cultural, respeto intergeneracional, igualdad de géneros. Consideramos importante ampliar la mirada del concepto de sostenibilidad y para ello analizamos e interpretamos espacios cotidianos. Es decir, queremos hacer un ejercicio de concreción del concepto de sostenibilidad (básicamente partiendo de los aspectos sociales) en el barrio.

Partimos de la idea que todo espacio público sostenible será aquel que proporcione calidad de vida tanto a la vecindad como a los paseantes. No son pocos los autores que han analizado y teorizado sobre cuáles pueden ser los indicadores objetivos para medir la calidad de vida de las ciudades y más concretamente de los espacios públicos (Holden, 2006; Alguacil, 2008; Ballas, 2013). La organización Project for Public Spaces² describió en 2002 (y en traducción al español de 2016) las cuatro cualidades indispensables que debía tener todo espacio público: 1) que fuera un espacio sociable, 2) polivalente en cuanto a usos y actividades que se pueden llevar a cabo, 3) confortable, visualmente atractivo, con valor histórico, 4) bien comunicado y accesible.

${ }^{2}$ El Trabajo de Project Public Space ha sido traducido al español por el organismo Plataforma Urbana. (2016). Se puede ver en la web citada en la bibliografía. 
Creemos que el espacio urbano se entenderá como un lugar sostenible y de calidad de vida siempre que pueda dar respuesta a las necesidades humanas del ciclo de la vida. Partiendo de las cualidades mencionadas, concretamos el análisis de la sostenibilidad de los barrios -y puede concretarse en plazas concretas- a través de siete conceptos clave: seguridad, sociabilidad, autonomía, identidad, accesibilidad, calidad y multifuncionalidad (Serra-Salvi, 2019):

1) Seguridad: se relaciona con la idea de permanecer en un lugar y desplazarse sintiendo confianza, sentirse cómodo, sin temer ser molestado/a por otras personas. También se añaden a este concepto, aspectos de visibilidad y señalización del espacio público.

2) Sociabilidad: detectar si el espacio favorece o no las relaciones sociales, las posibilidades de desarrollar actividades, de ser un punto de encuentro y de compartir el tiempo personal en un espacio público.

3) Autonomía: se refiere a si todo el mundo puede realizar sus actividades sin sentirse excluido. Se tienen en cuenta tanto los elementos que favorecen la autonomía, la libertad de movimiento y de acción, como los que pudieran perjudicarla.

4) Identidad: la podemos identificar con las opiniones, juicios y descripciones que se relacionan con el sentido de pertenencia a un barrio, de estima hacia el lugar vivido y de rasgos simbólicos compartidos en el seno de la comunidad.

5) Accesibilidad: nos referimos a todas las cuestiones que posibilitan o dificultan (incluso impiden) la llegada y el desplazamiento interior en cualquier espacio público. Se observan concretamente: transporte público, zonas de aparcamientos de todo tipo de vehículos de motor y bicicletas, posibles barreras arquitectónicas que impidan el acceso para colectivos de personas.

6) Calidad: la relacionamos con la confortabilidad del espacio público, en el sentido de si es agradable pasear y/o permanecer en él, si es limpio, si tiene zonas verdes, si dispone de bancos para el descanso, si es percibido como "zona agradable".

7) Multifuncionalidad: o la presencia de servicios complementarios que cubren necesidades o gustos de los transeúntes. Por ejemplo, la presencia de comercios, equipamientos lúdicos, sociales, deportivos y de salud para todos los colectivos, espacios de juego y puntos de encuentro.

\subsection{La ciudad que quiero}

La cuarta actividad es tan sencilla como una corta redacción (máximo diez líneas) acerca de cómo debe ser la ciudad ideal de cada estudiante. Pedimos esta tarea una vez completadas las actividades anteriores, y el análisis de estos pequeños textos nos 
sirven para saber qué ideas y conceptos de los anteriormente tratados han quedado en la mente de los estudiantes y los consideran importantes para mencionarlos. Veremos, a continuación, los resultados.

\section{RESULTADOS}

\section{1. ¿Cómo dibujan el mapa mental de la ciudad?}

Hemos analizado una muestra de treinta dibujos (de ciento treinta posibles), mitad hombres y mitad mujeres. Podemos observar los elementos establecidos por Kevin Lynch (1960): las sendas (paths), las fronteras entre distintos lugares (edge), algunos barrios (district), puntos estratégicos (nodes) que conectan distintos lugares de la ciudad y los hitos (landmarks), señales de referencia que facilitan la orientación.

Observando los dibujos y desde una perspectiva de género, vale la pena destacar algunas diferencias. Los dibujos de los chicos, a la vez que en conjunto describen menos detalles, relatan una mirada más amplia de la ciudad, en la que los nodos (representados por las rotondas) tienen un papel destacado. Sus dibujos tienden a caracterizarse por un número mayor de líneas rectas y cuadrículas, en las que las calles y los conjuntos de casas se van encontrando. En sus dibujos, los chicos anotan muchas más indicaciones hacia distintas direcciones y barrios externos, que en el caso de las chicas.

Las ilustraciones de las mujeres, por otro lado, son más ricos en matices y puntos de referencia. Por lo general describen un espacio reducido y concreto de la ciudad. Es en la observación de servicios culturales y religiosos donde recae una mayor diferencia a nivel de género. Las iglesias, las escuelas, los centros culturales, las bibliotecas y los edificios emblemáticos del lugar son mucho más localizados en los dibujos de las chicas. También, los espacios públicos, como plazas y parques, aparecen en más dibujos de chicas, mientras que en el caso de los chicos estos se esbozan en pocas ilustraciones. Podemos afirmar, sin lugar a dudas, que en el mapa mental de las chicas los espacios públicos juegan un papel más significativo que en el de los chicos. En el mismo sentido, las mujeres tienen más conciencia de los servicios de salud (centros de atención primaria, farmacias) así como de las tiendas y los comercios.

Al mayor nivel de detalle ya descrito de los bocetos de las mujeres, vale la pena observar que son ellas quienes identifican más las calles con sus nombres. Igualmente, también son ellas las que distinguen más elementos de detalle como árboles, personas y elementos curiosos. En relación a la descripción de elementos relacionados con los medios de transporte (estaciones de bus y tren), equipamientos deportivos y espacios naturales, no se dan diferencias de género especialmente significativas. A modo de ejemplo, podemos observar el mapa mental de la Figura 1. 


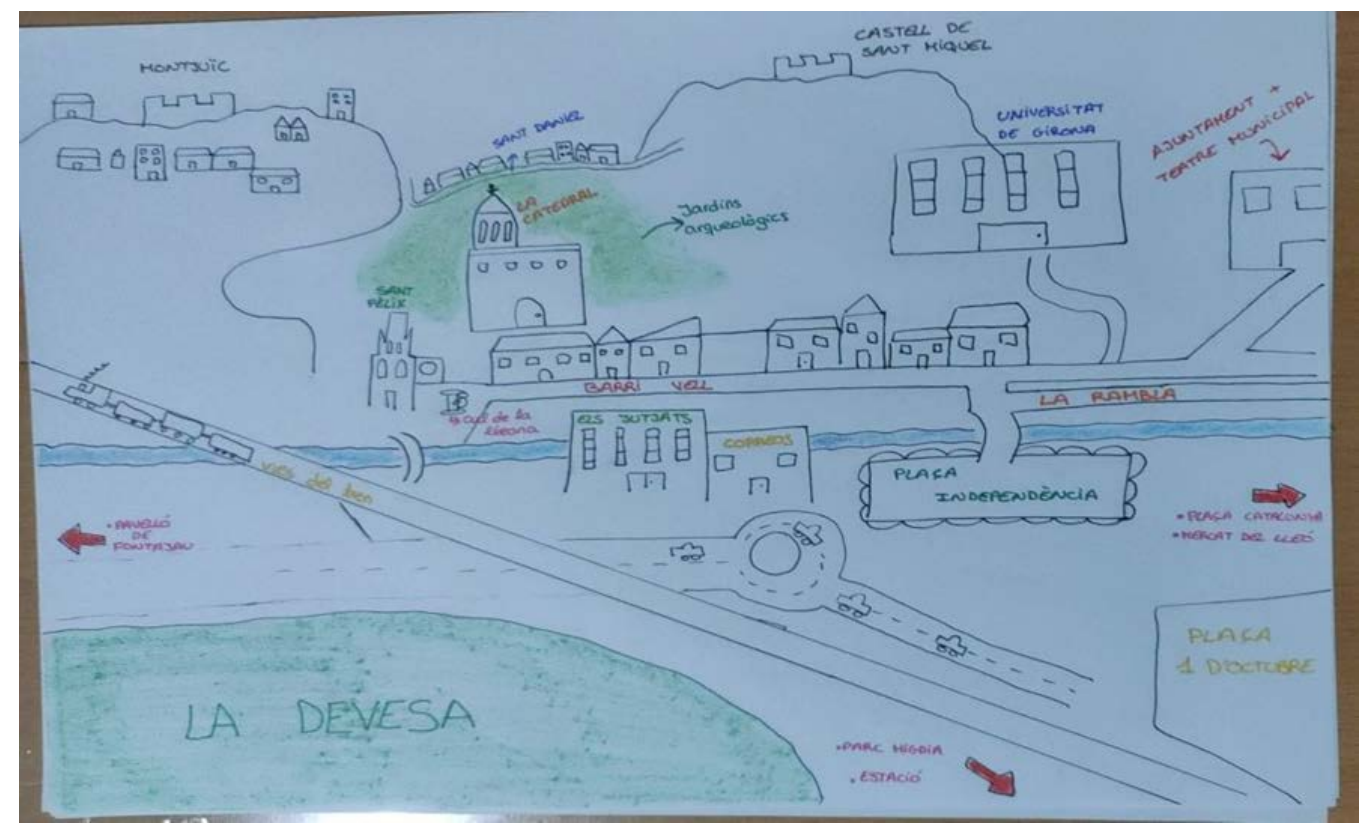

Figura 1. Mapa mental de la ciudad de Girona

\section{2 ¿Cómo interpretan la ciudad en clave de género?}

\subsubsection{Espacio público y micro violencias de género}

En esta actividad hemos unido el ODS 5 de Igualdad de género con el ODS 11 de Ciudades y comunidades sostenibles. La actividad de aula a través del blog preparado por las profesoras (https://ciutatigenere.blogspot.com) trata el tema de la posible violencia hacia las mujeres en las ciudades en formas muy diversas, a menudo, poco perceptibles. Los y las estudiantes, se plantean, al inicio, si se han percatado o no de ello. A continuación, con los textos y las filmaciones que hemos recogido en el blog, nos adentramos en esta cuestión.

En la encuesta inicial, la totalidad del alumnado reconoce que la violencia es una realidad que se muestra de maneras muy distintas. Haciendo atención al espacio público, de las 128 respuestas obtenidas el 82' $\%$ asiente que el entorno ejerce opresión hacia las mujeres, mientras que el $5 \%$ considera lo contrario y el 16'5\% afirma no haberse planteado nunca esta cuestión. Al finalizar la actividad, el 96'8\% reconoce que el espacio ejerce opresión a las mujeres mientras que un $4 \%$ mantiene que esa realidad no se da. 
Así mismo, ante la petición de propuestas para construir una ciudad más igualitaria encontramos dos tipos de respuestas. Unas, son aportaciones generales que no encuentran concreción (a modo de ejemplo: que los chicos no digan cosas a las chicas si estas van solas, eliminar los comentarios machistas, mejorar la justicia y las leyes...) Otras, concretas, consideran que es necesario trabajar la educación en valores desde la escuela (22\%) así como mejorar la seguridad nocturna (6,25\%, a nivel general) y dotar de cámaras y mejor iluminación las calles $(3,5 \%)$.

Realizadas las actividades requeridas en el blog, las preguntas son contestadas con mucho más matiz en relación al tema urbano. La opresión contra las mujeres más allá de las agresiones físicas (15\%) y verbales (27\%) y ahora aparecen observaciones que al inicio no aparecían: se contempla la falta de seguridad sobre todo de noche $(4,8 \%)$, el uso diferenciado por razón de género del transporte público (15\%), los nombres de las calles con personajes masculinos $(5,6 \%)$, los anuncios y carteles con mensajes machistas $(8 \%)$, entre otros.

Así mismo, en la encuesta final, los estudiantes apuntan ideas para construir una ciudad igualitaria. Más allá de la educación de la ciudadanía que desempeña un valor relevante y esencial (22\%), la intervención en el entorno urbano adquiere un papel fundamental. El $14 \%$ opina que es importante invertir en la seguridad mayormente por la noche. Se afirma la necesidad de construir calles anchas $(4,2 \%)$, con buena iluminación (27\%), más protección, cámaras $(14 \%)$ y vigilancia policial $(6,9 \%)$. En menor medida se hace atención a la dotación de transporte público especialmente de noche (5,3\%), a la presencia de puntos violetas (3\%) así como de normas y leyes a favor de las mujeres (3\%).

Creemos que la práctica con el blog ha permitido alcanzar aprendizajes aplicados y de relevancia a nivel local con repercusión a nivel global. Así mismo, hemos conseguido introducir la perspectiva género en la lectura de la ciudad facilitando así una participación crítica del alumnado en la toma de decisiones de sus pueblos y ciudades.

A continuación, y fuera del aula, los estudiantes llevan a cabo un trabajo de campo para la observación en la ciudad de: 1) la representación de la mujer en el espacio urbano y 2) los roles de hombres y mujeres en actividades cotidianas en la ciudad.

\subsubsection{Sobre la representación de la mujer en el espacio urbano}

Se han analizado de forma pormenorizado una muestra de trabajos realizados por los y las estudiantes que implican diez grupos de trabajo, que significa unos cincuenta estudiantes.

La representación de la mujer más observada es la mujer como objeto de deseo sexual que responde a unos cánones de belleza. Dicha representación se ha recogido principalmente de los anuncios publicitarios de productos de belleza en escaparates de 
centros de estética, oficinas de farmacia y tiendas de ropa íntima, principalmente. La representación que ocupa un segundo lugar por número de observaciones es el de la mujer asumiendo el rol de madre feliz y servicial al servicio de la familia. La mayoría de las veces se trata de carteles en los que se ve la mujer abrazando o atendiendo los niños, desde ámbitos tan diversos como los anuncios de juegos, de cremas hidratantes o de seguros de vida. En este grupo, se incluye la imaginería religiosa católica. Nuestros estudiantes tienden a interpretar en las imágenes de las vírgenes de la religión católica, la concepción de una mujer sumisa.

Al lado de estas representaciones clásicas de la mujer en una sociedad patriarcal, el estudiantado ha encontrado una conceptualización de la mujer empoderada. Una mujer que se presenta reivindicativa hacia una igualdad de género, por un lado, a la vez que denuncia las agresiones y los maltratos, por otro. Muchas de estas ilustraciones se encuentran en carteles violetas, grafitis y murales de pared. Ocupan en su mayoría, espacios ya de por si alternativos (debajo de puentes, vías de tren, paredes de edificios abandonados, cercas, muros...).

En menor cantidad, pero no por ello menos importante, se ha observado la mujer como símbolo de naturaleza, relacionado con su capacidad de dar vida, a la vez que como personificación del trabajo y el esfuerzo. También, la mujer como figura de cultura y creatividad, resultado de la voluntad de visibilizar los trabajos artísticos y literarios que durante siglos se han menospreciado. Dichas representaciones se encuentran en carteles divulgativos y en los nombres otorgados a centros culturales. Pocas imágenes han recogido el simbolismo de la inocencia. Dichas representaciones de la mujer, contrastan con los anuncios de hombres que principalmente encontramos en anuncios de bancos, deportes y trabajo.

\subsubsection{Roles de género en la ciudad}

Finalmente, pedimos a los estudiantes observar en diferentes espacios de la ciudad qué hacen los hombres y qué hacen las mujeres. En los supermercados, se observaron muchas más mujeres que hombres comprando, el mismo resultado en los puestos de los mercados municipales. Las lecturas de los estudiantes son ricas en matices: han visto que muchas mujeres van solas a la compra; si van solas, compran bastante; pero también es habitual verlas acompañadas de sus hijos o hijas de corta edad, en este caso, las compras son menores. También han observado que los hombres suelen ir solos a los establecimientos, compran menos, o bien, acompañan a las mujeres, las cuáles suelen dirigir la actividad de la compra. En los espacios de cafeterías o bares, se observaron más frecuentados por hombres que por mujeres. Ellos van solos; ellas van acompañadas por otras mujeres o por un hombre, se supone su pareja. En los espacios frente a un centro 
escolar, la presencia femenina se ha detectado con fuerza, mayor que la masculina. Los hombres utilizan, casi todos, el vehículo propio para recoger o dejar a los hijos e hijas; en cambio, las mujeres, se las ve utilizar -algo más- el transporte público o ir andando. También se observó quienes hacen uso de las plazas de la ciudad. La presencia de hombres y mujeres es bastante similar; sus actividades difieren: las mujeres están atentas a los juegos de los niños y niñas; los hombres charlan en tertulias, sentados en terrazas de bares o jugando a la petanca. Acerca del transporte público, se ha detectado que las mujeres son mucho más usuarias que los hombres, prácticamente en todas las líneas urbanas de la ciudad, solas o acompañadas de los hijos o hijas de corta edad.

\section{3. ¿Cómo reconocen y valoran el espacio público de la ciudad?}

Los y las estudiantes, organizados en grupos de trabajo, observan y analizan una zona de la ciudad con la guía de los siete conceptos clave antes mencionados. Nuestros estudiantes de primer curso conocen poco la ciudad de Girona, que les acoge como ciudad universitaria, pero en la cual muchos no residen, ya que los movimientos pendulares diarios son muy usuales. Por tanto, para la mayoría, ir a pasear y observar un barrio de la ciudad es una novedad, sobre todo si no es el centro. Al igual que en la temática anterior, hemos analizado de forma más pormenorizada diez grupos de trabajo, que implica a unos cincuenta estudiantes.

Realizan un reportaje informativo, con mucha imagen, con el hilo conductor de los siete conceptos clave. Observamos las siguientes características en sus trabajos:

- Uno de los temas que más les preocupa es el de la seguridad, sentirse seguro/a en el barrio o en la plaza. Es un tema del que hablan con reiteración, uniéndolo a la organización del tráfico rodado y a la iluminación.

- Se fijan en la sociabilidad, básicamente en los espacios verdes y plazas, comentan si ello es posible en estos espacios y poco más. Hemos constatado que muchos grupos no asocian la sociabilidad a los equipamientos de barrio como centros cívicos, es como si no conocieran las funciones de estos centros.

- Los conceptos de autonomía y de accesibilidad son vistos como uno solo por la mayoría de grupos de trabajo. La accesibilidad con el transporte privado (coches), y la ausencia o pocas plazas de parking sin pagar son preocupaciones evidentes de los estudiantes. De todas formas, saben que "deben valorar" los transportes colectivos y la bicicleta en la ciudad, y lo hacen. Pero en su discurso, detectamos una preocupación generalizada y reiterada por las plazas de parking de los coches.

- El desconocimiento inicial de los barrios provoca que, en algunos casos, se profundice muy poco o nada en el sentido de identidad del barrio. En otros casos, se descubre, de forma positiva. Unos grupos lo consiguen, y otros no. 
- Las zonas verdes son las más reiteradamente valoradas, incluso cuando son exageradamente pequeñas para el ámbito poblacional.

- En general, tienden a valorar en exceso la positividad de la mayoría de los conceptos (excepto algunos casos muy evidentes con problemas) y a concluir, por tanto, que la ciudad está en buena dirección en relación a más sostenibilidad en el ámbito social.

- Constatamos la formulación de buenas preguntas entre los grupos de trabajo ante la realidad urbana observada.

- Aprenden conocimientos de geografía urbana a través de un proceso de aprendizaje aplicado, directamente en el barrio objeto de estudio

- Aparecen las dudas acerca de los propios valores asumidos como ideas previas ante la realidad urbana.

En definitiva, pensamos que el trabajo es positivo porque les obliga a mirar a la ciudad desde diferentes puntos de vista que hasta ahora no se habían planteado y que les han de servir para el estudio de la ciudad con posibles alumnos de primaria, en su futura vida profesional.

\section{4. ¿Cómo es su ciudad ideal?}

Recordamos que los y las estudiantes redactan una pequeña redacción describiendo su ciudad ideal, como final del proceso de la docencia y trabajo de campo referido al estudio urbano.

Cuando el estudiantado describe la ciudad ideal, mayormente coincide, sin diferencias de género destacables, en imaginarla como un lugar con buenos accesos e inclusiva. Hacen especial atención a que garantice calidad de vida a todos los colectivos y especialmente de aquellos que se encuentran en una situación de vulnerabilidad por su condición de diversidad funcional. Piensan en buenos medios de transporte público y redes de comunicación que conecten los distintos barrios de la ciudad.

La calidad de vida en la ciudad es otro de los valores más considerados al describir la ciudad preferida. Dicha calidad se concibe a partir de disponer de espacios verdes que permitan el contacto con la naturaleza y sin contaminación. En los chicos, la preferencia por los espacios verdes es ligeramente superior que en las chicas. Vale la pena destacar que los hombres piensan en un espacio verde para el deporte, aspecto no mencionado por sus compañeras. Por otro lado, las chicas (73\% frente, el $47 \%$ de los chicos) ponen el acento a su preferencia por un lugar sin contaminación, con el aire puro. También son 
las chicas las que hacen mayor hincapié (en un 60\%) en el deseo de vivir en un lugar que sea bonito, agradable y limpio.

La calidad de vida de una ciudad se procesa también a partir de la cultura de la dotación de equipamientos que incentiven la actividad cultural (como teatros, cines, museos...) así como a través del gozo de la libertad de expresión y de movimiento. Un deseo en la ciudad ideal manifestado en un $13 \%$ de los y las estudiantes.

En la descripción de la ciudad ideal el 53\% están de acuerdo en que dicha ciudad debe cubrir las necesidades básicas de todas las personas para garantizar una vida digna y con igualdad de oportunidades. Entienden que en ella no debería haber quien pasara hambre o durmiese en la calle. Ante las personas en situación de vulnerabilidad, las chicas (67\%) son especialmente más sensibles que sus compañeros (40\%).

Una ciudad multifuncional es descrita a partir de una buena dotación de servicios (comercios, centros educativos, de salud, lúdicos...) repartidos por la ciudad, en un $27 \%$ de los chicos, respecto a $20 \%$ de las chicas. También son los chicos (27\% frente el 13\% de las chicas) los que mayormente esperan que la ciudad ideal asegure un buen acceso a los servicios básicos.

Otro de los aspectos más considerados al imaginar la ciudad ideal es la seguridad (53\%). Se entiende por seguridad poder desplazarse de manera cómoda, sin miedo, con autonomía de día y de noche, sentirse a gusto. En el diseño urbano dicha seguridad se traduce en más iluminación a fin de evitar intimidaciones y robos a opinión de las chicas, en el caso de los chicos supone un paso imprescindible para garantizar una mejor seguridad viaria. En su conjunto, el tema de la seguridad es de mayor preocupación para las chicas (67\%) que para los chicos (40\%). Relacionado a este concepto encontramos el anhelo de vivir en un entorno de paz, tranquilidad y armonía (20\%).

La ciudad ideal se percibe sin especiales distinciones entre chicos y chicas, como lugar donde no haya discriminaciones por motivos de género (30\%). Hombres, mujeres y personas de género no binario se sienten representados con equidad. Un tratamiento igualitario (17\%), respetuoso con la diversidad cultural, de creencias religiosas y origen étnico, ha sido considerado solamente por los chicos. Otra característica considerada en la imaginación de la ciudad perfecta se vincula a la participación y la identidad (en un 23\%) sin notables diferencias de género.

Un último atributo que aparece en el retrato del espacio urbano preferido es la sociabilidad (en un 23\%), con una notable distinción de género. Las chicas (un $33 \%$ respeto a un $13 \%$ de los chicos), ponen de relieve la importancia que la ciudad propicie el encuentro y la cohesión social. 
Podemos considerar que nuestro estudiantado ha consolidado el aprendizaje del concepto de sostenibilidad relacionado al concepto de ciudad o espacio urbano. En sus descripciones, se relatan características que de una forma $\mathrm{u}$ otra coinciden con los conceptos claves presentados, que responden a las necesidades de la ciudadanía y le garantizan calidad de vida.

\section{CONCLUSIONES}

Al iniciar la asignatura, en las encuestas de evaluación inicial, el término Sostenibilidad viene asociado a los problemas en relación con el medio ambiente, tales como contaminación, energía, agua, residuos, cambio climático (y, por tanto, con los ODS que los tratan). Nuestro empeño, en esta asignatura, es conseguir que al término Sostenibilidad se asocien también los problemas (y ODS) más sociales tales cómo igualdad de género, calidad de vida en las ciudades, urbanismo amable para todas las funciones urbanas y para la diversidad de personas, trabajo decente, comercio justo y de proximidad, finanzas éticas, educación para la paz. En este artículo hemos tratado los temas relacionados con la ciudad.

El desarrollo de las distintas actividades expuestas, las miradas urbanas que tenemos la oportunidad de llevar a cabo, creemos que ha hecho cambiar las concepciones iniciales de nuestros estudiantes acerca de la ciudad. Con los dibujos mentales se percatan de las dificultades de traspasar en un papel su idea de la ciudad que conocen (y aún más difícil si les pedimos que todos ellos dibujen la ciudad donde estudian porque la mayoría la conocen poco). Conocer la ciudad sería el primer paso para pensar como la queremos, desde una óptica de ciudadanos responsables y futuros docentes.

Observar la ciudad en clave de género es una novedad para nuestros estudiantes. La mayoría de ellos y ellas reconocen no haberse percatado antes de la importancia del urbanismo "amable" para las actividades de hombres y mujeres y obtener una mayor calidad de vida en una ciudad mediana, con densidades de población importantes en algunas zonas. Las diferentes actividades de los hombres y de las mujeres en la ciudad son reconocidas como roles género heredados de una cultura patriarcal y los y las estudiantes se plantean como aún hoy se perpetúan.

El espacio público, a través de la observación de plazas y calles, dando respuesta a la lista de los siete conceptos clave enunciados anteriormente dan la línea a seguir para conseguir una ciudad más sostenible desde la parte más social de la sostenibilidad. Los y las estudiantes se fijan en ellos, se plantean preguntas y se posicionan acerca de cómo mejorar la ciudad. El proceso, además, es una guía para el "descubrimiento" de los barrios y del tipo de vida que la población lleva en ellos. 
Finalmente, una pequeña redacción sintetiza todo aquello que los estudiantes han incorporado en su "mirada urbana". Observamos que las actividades de los hombres y de las mujeres son más importantes en su concepto de ciudad. Vemos una mirada más crítica al funcionamiento de la ciudad actual. Constatamos que el concepto de sostenibilidad se ha ampliado de forma consistente, de únicamente "lo verde" a la calidad de vida.

\section{REFERENCIAS BIBLIOGRÁFICAS}

Alguacil, J. (2008). Espacio público y espacio político: la ciudad como lugar para las estrategias participativas. Boletín $C F+S, 44,51-65$ http://habitat.aq.upm.es/ boletin/n44/ajalg2.html

Asociación de Geografía de España (2019). Declaración conjunta del Colegio de Geógrafos de España y de la Asociación Española de Geografía (AGE) ante la propuesta de incluir una asignatura de "cambio climático" en las enseñanzas universitarias de nuestro país. https://www.geografos.org/declaracion-conjuntacolegio-de-geografos-age/

Ballas, D. (2013). What makes a happy city?. Cities. The International Journal of Urban Policy and Planning, 32, S39-S50 https://doi.org/10.1016/j.cities.2013.04.009

Bofill, A. (1993). Urbanisme i gènere. L'urbanisme des de la política de les dones. Barcelona Societat, 19, 76-86 https://ajuntament.barcelona.cat/dretssocials/sites/ default/files/revista/revista-19-art6-urbanisme-i-genere-urbanisme-politiquesdones.pdf

Borja, J. (2013). Revolución urbana y derechos ciudadanos. Alianza Editorial

Collazo, L.M. y Granados Sánchez, J. (2020). Implementation of SDGs in University Teaching: A Course for Professional Development of Teachers in Education for Sustainability for a Transformative Action, Sustainability, 12(19), 8267 https:// doi.org/10.3390/su12198267

Corrales, M., Sánchez, J., Moreno, J. y Zamora, F. (2020). Educar en la sostenibilidad: retos y posibilidades para la didàctica de las ciencias sociales. Cuadernos de Investigación en Juventud, 8, 36-59 https://doi.org/10.22400/cij.8.e046

Corrales, M. y Garrido, J. (2021). Los objetivos de desarrollo sostenible como contenido transversal en la enseñanza de la Geografía. Una experiència de gamificación en $1^{\circ}$ de ESO. Didacticae, 9, 7-23 https://doi.org/10.1344/did.2021.9

De Miguel González, R. (2018). Geografía y tiempo contemporáneo: educación geogràfica y enseñanza de las ciencias sociales para el mundo global. REIDICS. Revista de Investigación en Didáctica de las Ciencias Sociales, 2, 36-54 https:// doi.org/10.17398/2531-0968.02.36 
De Miguel González, R. (2020). From international to global understanding: toward a century of international geography education, International Research in Geographical and Environmental Education https://doi.org/10.1080/10382046.2 $\underline{020.1809792}$

Granados Sánchez, J. (2010). La educación para la sostenibilidad en la enseñanza de la geografía. Un estudio de caso. Enseñanza de las Ciencias Sociales 10, 29-41 https://www.raco.cat/index.php/EnsenanzaCS/article/view/248890/333136

Holden, M. (2006). Urban indicators and the integrative ideals of cities. Cities. The International Journal of Urban Policy and Planning ,23(3), 170-183 https://doi. org/10.1016/j.cities.2006.03.001170

Khusnutdinova, S., Rodionova, I. y Adashova, T. (2019). The mental maps "the image of the city" in the teaching process of the discipline "urban geography" International Multidisciplinary Scientific GeoConference Surveying Geology and Mining Ecology Management, SGEM, 19(5.4), 327-332 https://doi.org/105593/ sgem2019/5.4/S22.044

Lefebvre, H. (1978). De lo rural a lo urbano. Ediciones Península.

Lynch, K. (1960; 2015 3a edición). La imagen de la ciudad. Gustavo Gili.

Haubrich, H., Reinfried, S. y Schleicher, Y. (2007). Declaración de Lucerna sobre Educación Geográfica para el Desarrollo Sostenible https://www.igu-cge.org/ wp-content/uploads/2018/02/spanish.pdf

IGU (2016). Declaración Internacional sobre Educación Geográfica https://www.igucge.org/wp-content/uploads/2019/08/IGU 2016 spanish updated.pdf

Ortiz, A., Garcia Ramon, M.D., y Prats, M. (2014). La Vila Júlia de Nou Barris: un estudio cualitativo y de género de un espacio público en Barcelona. En M.D. Garcia Ramon, A. Ortiz y M. Prats (Eds) Espacios públicos, género y diversidad: Geografías para unas ciudades inclusivas (p.61-76). Barcelona: Icaria.

Plataforma Urbana (2016). 6 lugares destacados por PPS como buenos ejemplos de espacios públicos. https://www.plataformaurbana.cl/archive/2016/05/09/6lugares-destacados-por-pps-como-buenos-ejemplos-de-espacios-publicos/

Serra-Salvi, A., y Medir, R.M. (2021). Enseñar a leer la ciudad en clave de género como punto de partida para una ciudadanía proactiva. En J. Granados y R.M. Medir (Eds.) Enseñar y aprender geografía para un mundo sostenible (p.251-260). Barcelona: Octaedro.

Serra-Salvi,A. (2019). Vida quotidiana, espai públic i construcció de sentit de pertinença a Girona. Una anàlisi intergeneracional, interseccional i de génere. (Tesi doctoral, Universitat de Girona, Catalunya). https://hdl.handle.net/10803/669783 
Tello, R. (2002). Mirades a l'espai públic: introducció. En R. Tello (Ed.) Espais públics: mirades multidisciplinàries. Barcelona: Pòrtic.

Tilbury, D. (2011) Educación para el desarrollo sostenible. Examen por los expertos de los procesos y el aprendizaje. ED-2010/WS/46. Paris: UNESCO https://unesdoc. unesco.org/ark:/48223/pf0000191442 spa?posInSet=8\&queryId=4cb49144877b-4121-8cdf-aa3fb580db97

Vilches, A. y Gil,D. (2012). La educación para la sostenibilidad en la Universidad: el reto de la formación del profesorado. Profesorado. Revista de Currículum y Formación de Profesorado, 16(2), 25-43. https://www.redalyc.org/pdf/567/56724395003.pdf 